\title{
Entre théorie et fiction : quelques figures paradoxales de l'Auteur dans Si par une nuit d'hiver un voyageur d'Italo Calvino
}

Anne-Marie Monluçon

\section{OpenEdition Journals}

Édition électronique

URL : http://journals.openedition.org/recherchestravaux/1287

DOI : 10.4000/recherchestravaux.1287

ISSN : 1969-6434

Éditeur

UGA Éditions/Université Grenoble Alpes

\section{Édition imprimée}

Date de publication : 15 mai 2004

Pagination : 141-156

ISBN : 2-9518254-3-9

ISSN : 0151-1874

Référence électronique

Anne-Marie Monluçon, «Entre théorie et fiction : quelques figures paradoxales de l'Auteur dans Si par une nuit d'hiver un voyageur d'Italo Calvino ", Recherches \& Travaux [En ligne], 64 | 2004, mis en ligne le 20 mai 2019, consulté le 10 décembre 2020. URL : http://journals.openedition.org/recherchestravaux/ 1287 ; DOI : https://doi.org/10.4000/recherchestravaux.1287 
Anne-Marie MONLUÇON

Université Stendhal-Grenoble 3

\section{Entre théorie et fiction}

\section{Quelques figures paradoxales de l'Auteur dans Si par une nuit d'hiver un voyageur d'Italo Calvino}

Le roman d'Italo Calvino, Si par une nuit d'biver un voyageur', se caractérise par une prolifération de figures paradoxales de l'auteur. Le personnage principal, appelé "Lecteur», mène une quête, au cours de laquelle il poursuit un traducteur faussaire, Hermès Marana, et un écrivain tourmenté, en panne d'inspiration, tenté par la vocation de copiste et peut-être par celle de plagiaire, Silas Flannery. La date du roman, ainsi que le parcours intellectuel de Calvino, nous porte à établir un lien: la multiplication des figures paradoxales de l'Auteur dans le roman n'est peut-être pas sans rapport avec le débat théorique qui l'a précédé de dix ans, au sujet de «la mort de l'Auteur ". Ce débat a été principalement lancé par un article de Roland Barthes, "La mort de l'auteur ${ }^{2} "$, paru en 1968 , et une conférence de Michel Foucault, "Qu'est-ce qu'un auteur?3", datant de 1969. Cependant, lier la fiction d'Italo Calvino aux théories des structuralistes français que sont Roland Barthes et Michel Foucault ne va pas de soi, car la critique a déjà bien établi que l'influence de Borges ${ }^{4}$ ou de l'Oulipo ${ }^{5}$ sur Calvino fut bien plus déter-

I. Italo Calvino, Si par une nuit d'biver un voyageur, (1979), trad. de l'italien par Danièle Sallenave et François Wahl, (1981), Seuil, coll. "Points. Roman», éd. de 1995.

2. Roland Barthes, "La mort de l'auteur" (1968), Essais critiques $I V$. Le bruissement de la langue, Seuil, 1984, p. 6I-67.

3. Michel Foucault, "Qu'est-ce qu'un auteur?" (1969), Dits et écrits (1954-1969), T. I, Gallimard, NRF, 1994, p. 789-82I.

4. Michel Lafon, "Borges, Calvino et la littérature", Actes du Colloque international "Borges, Calvino, La littérature", Madrid, Fundamentos, 1996, vol. I, p. II-25.

5. Marcel Benabou, "Si par une nuit d'hiver un oulipien... ", Magazine litteraire, $n^{\circ}{ }_{274}$, février 1990, P. 4I-43. 
minante que celle des structuralistes. Pourtant, trois arguments autorisent un tel rapprochement. Le premier est d'ordre biographique - si l'on ose avancer un argument aussi sacrilège, à propos du débat sur la mort de l'auteur -: Italo Calvino a résidé à Paris de 1967 à 1980 et l'on sait qu'à cette époque il a lu, et même, pour certains d'entre eux, fréquenté personnellement les théoriciens de la mort de l'auteur. En second lieu - argument bibliographique - la critique, principalement anglo-saxonne, a consacré des études aux rapports entre les théories barthésiennes et la fiction calvinienne ${ }^{6}$. Mais elle s'est essentiellement limitée à considérer Italo Calvino comme un lecteur docile de Roland Barthes, en négligeant les références foucaldiennes et en centrant la réflexion sur le couple auteur-lecteur ou narrateur-narrataire, sans prendre en compte les figures du faussaire, du plagiaire ou du copiste. Enfin, un argument intertextuel. On trouve non seulement l'illustration des idées de Roland Barthes et de Michel Foucault dans la structure de Si par une nuit d'hiver un voyageur, mais également des citations ou paraphrases des deux célèbres articles de 1968 et 1969. Les raisons biographiques et bibliographiques que l'on a de confronter le roman aux deux articles étant bien connues, l'analyse intertextuelle retiendra seule notre attention ici.

Chercher à savoir si Calvino est d'accord avec les positions, sensiblement différentes au demeurant, de Roland Barthes et Michel Foucault 7 , et comment son opinion transparaît dans le roman, ne présente sans doute qu'un intérêt anecdotique. Le véritable enjeu se situe plutôt au niveau des écarts entre la théorie et ce qu'en fait la fiction. Si c'est un Auteur, et non plus un critique, qui défend la thèse de la "mort de l'Auteur", quelle incidence a ce changement de point de vue? L'écrivain se distingue d'abord par les moyens qu'il choisit pour s'exprimer, et, notamment, par le recours à la fiction. Or, la fiction confere au romancier une liberté souveraine, grâce à laquelle il ne se contente pas de reprendre à son compte l'hypothèse de la mort de l'Auteur comme principe de lecture - ou comment se passer de l'intention de l'auteur et de sa biographie pour lire? Elle permet aussi de se demander qui reste pour

6. Melissa Watts, «Reinscribing a dead Author in If on a Winter's Night a Traveler", Modern Fiction Studies, Baltimore, MD (MES), 1991 Winter 37: 4, p. 705-716, et Patricia Waugh, Metafiction, London, Methuen, 1984 .

7. La différence la plus évidente entre l'article de Roland Barthes et la conférence de Michel Foucault réside dans le ton, curieusement moins idéologique et révolutionnaire chez le second. En second lieu, la corrélation de la mort de l'Auteur avec la naissance du lecteur est une idée propre à Barthes, et totalement absente chez Foucault. Enfin, le philosophe dénonce l'écriture comme le remplacement d'une sacralisation - celle de l'Auteur - par une autre, et l'on peut se demander s'il ne s'agit pas d'une polémique avec Roland Barthes, qu'il se garde cependant de nommer. 
écrire, une fois l'Auteur mort? À l'horizon de son roman, surgit l'idée d'une littérature de faussaire, qui semble obtenir curieusement l'assentiment de l'Auteur. En d'autres termes, le romancier interroge l'hypothèse de la mort de l'auteur comme principe d'écriture, de production des textes.

\section{Calvino et les théoriciens français de la «mort de l'Auteur"}

Il est tentant de rapprocher le roman des articles de Roland Barthes et Michel Foucault, à deux niveaux. D'abord, la structure du récit nous fait expérimenter la mort de l'Auteur, ce qui est bien différent de ce que serait une simple thématisation du sujet. En second lieu, la présence de citations et de paraphrases des deux articles dans le roman permet d'affirmer l'existence d'une intertextualité.

En ce qui concerne la structure, la critique a fait du système d'énonciation son principal motif de rapprochement avec l'article de Barthes ${ }^{8}$. On y a vu l'illustration du recentrage du phénomène littéraire autour du lecteur, que Roland Barthes appelle de ses vœux: "Nous savons que, pour rendre à l'écriture son avenir, il faut en renverser le mythe: la naissance du lecteur doit se payer de la mort de l'Auteur" (R. B., p. 67). Or, le récit à la deuxième personne qu'adopte Italo Calvino ne produit qu'un mirage de roman, centré sur le lecteur, dont nous serions le héros. Le narrataire étant désigné par le nom de Lecteur (avec un L majuscule), la tentation est grande de nous identifier à lui. Mais, au fur et à mesure que son portrait se précise, nous sommes contraints d'admettre que ce héros lecteur, le Narrataire, n'est pas nous, lecteurs réels, mais un personnage de lecteur ${ }^{9}$. Dans ce cas, la ressemblance du récit avec l'idée de Barthes n'est qu'un leurre, qui recouvre, en réalité, une véritable divergence. Non seulement le roman n'est pas centré sur le lecteur réel, mais ses nombreux passages réflexifs, dans lesquels le narrateur prodigue

8. D'après M. Watts (art. cit., p. 705), P. Waugh voit dans Si par une nuit d'hiver un voyageur un roman "de la naissance du lecteur, au prix de la mort de l'Auteur" (Waugh, p. 42, I34).

9. Philippe Daros (Italo Calvino: un itinéraire d'écriture dans la perspective des champs critiques contemporains italiens et français, 1988, Amiens, thèse non publiée, microfiche: 88 AMIE ooor) rend hommage à la clairvoyance pionnière de Carlo Segre (* Se una notte d'inverno uno scrittore sognasse un aleph di dieci colori *, Strumenti Critici, oct. 1979, II-III, p. 178-214) en rappelant qu'il a, l'un des premiers, souligné que le héros lecteur n'est pas le lecteur réel, et en traduisant la conclusion de Segre: "Il y a donc un lecteur 1, potentiel et absolument libre, et un lecteur 2, actuel, inventé et totalement hétéroguidé comme n'importe quel personnage de roman" (Daros, p. 467). 
au héros des conseils de lecture, ou se moque de lui, permettent, par ricochet, une impérieuse prise en main du lecteur réel, c'est-à-dire un retour en force de l'auteur Calvino dans son œuvre ${ }^{\mathrm{IO}}$.

Malgré sa fortune critique, ce choix énonciatif résiste moins à l'analyse que l'effet produit par la structure d'alternance et de quête. Douze chapitres numérotés, qui relatent l'histoire du héros lecteur, constituent ce que le narrateur appelle le "roman à vivre» (p. 38). Ce récit enchâssant raconte comment le héros ne trouve à lire que des romans incomplets, réduits à leur incipit. Son but est alors de se procurer à tout prix la suite, grâce à un exemplaire complet. Sa lecture est interrompue par une cause qui varie à chaque fois (erreur de brochage, d'impression, vol du livre, etc.). Ces interruptions se doublent d'une substitution. Chaque fois que le personnage croit avoir trouvé la suite du roman qu'il a commencé, il s'agit, en fait, de l'œuvre d'un autre auteur. Le personnage en vient donc à chercher non seulement la suite du roman, mais l'identité du véritable auteur du texte qu'il a en main. Or, l'une des hypothèses que fait Michel Foucault résonne comme une parfaite programmation de cette quête: "Et si, par suite d'un accident ou d'une volonté explicite de l'auteur, il [le texte] nous parvient dans l'anonymat, le jeu est aussitôt de retrouver l'auteur" (M.F., p. 80o). Le changement d'attribution est donc à la fois un thème du "roman à vivre" et l'expérience que nous fait faire l'alternance des douze chapitres racontant les aventures du héros avec les dix chapitres constitués par les débuts de romans qu'il lit et que le narrateur appelle les "romans à lire". Les cinq premiers "romans à lire" sont tous l'objet d'une attribution erronée, suivie d'une correction. La mécanique se grippe pour les sixième et septième "romans à lire". Le Lecteur se procure le sixième chez l'éditeur Cavedagna, qui le tient de Marana, et il trouve le septième chez son amie lectrice, Ludmilla, où il sait que Marana "volait des livres et revient maintenant les remplacer par des livres faux" (p. 178). Le Lecteur sait donc à l'avance que l'attribution des " romans à lire" 6 et 7 à Silas Flannery est peut-être fausse. En désespoir de cause, il redécouvre, avec Ludmilla, la "fonction auteur" de base et se rend en Suisse pour demander à Silas Flannery d'authentifier l'un de ces deux romans (p. 180). Or, ce dernier refuse de répondre à sa question (p. 216-217). En d'autres termes, cette séquence opère un saut qualitatif: nous passons d'un système de changement d'attribution à une absence d'attribution. C'est en ce sens que l'intrigue fait

Io. Ph. Daros, op. cit., p. $55 \mathrm{r}:$ : [...] ce roman se laisse analyser moins comme une fiction sur le désir de lecture que comme fiction qui suggère, coercitivement, la façon dont la lecture doit être menée [...] ", et p. 556: "Si par une nuit d'hiver un voyageur témoigne d'une prescription de lecture moins "participative" que "terroriste" ". 
écho à la formule de Roland Barthes: comme l'écriture, «elle détruit [toute voix], toute origine ". Mais plus profondément, elle mime l'avènement d'une "littérature anonyme", idée chère à J.L. Borges.

Si la trame du roman doit au moins autant à J.L. Borges qu'à l'influence des deux théoriciens français, en revanche, la lettre du texte porte plus sûrement leur empreinte, comme le révèle l'analyse intertextuelle. Ainsi plusieurs arguments contre la littérature traditionnelle et la conception ancienne de l'Auteur (garant de la vérité) se retrouvent théorisés, tantôt par le traducteur faussaire, Hermès Marana (p. II3-179-200), tantôt par l'écrivain Silas Flannery. La correspondance de Marana avec l'éditeur commence en ces termes:

Qu'importe le nom de l'auteur en couverture? Transportons-nous en pensée d'ici à trois mille ans. Dieu sait quels livres de notre époque auront survécu, et de quels auteurs on se rappellera encore le nom. Certains livres seront restés célèbres, mais on les considérera comme des œuvres anonymes, comme l'est pour nous l'épopée de Gilgamesh; il y aura des auteurs dont le nom sera demeuré célèbre, mais dont il ne restera aucune œuvre, comme c'est le cas pour Socrate; ou bien tous les livres qui auront survécu seront attribués à un mystérieux auteur unique, comme Homère (p. 113 ).

Cet incipit ressemble fort à une paraphrase du début de la conférence de Michel Foucault, où il cite Beckett: "QQu'importe qui parle?" En cette indifférence s'affirme le principe éthique, le plus fondamental peut-être, de l'écriture contemporaine» (M.F., p. 789). Le jeu d'écho ne doit cependant pas masquer une différence de fond, puisque Foucault, en citant Beckett et en affirmant qu'un changement d'attribution n'est pas sans conséquence, prend ses distances par rapport à cette indifférence, tandis que Marana souscrit sans réserve à sa thèse. Plus loin, le faussaire semble se souvenir de la "fonction auteur" foucaldienne. Le philosophe écrivait: "Qu'est-ce que cette règle de la disparition de l'écrivain ou de l'auteur permet de découvrir? Elle permet de découvrir le jeu de la fonction auteur" (M.F., p. 817). Marana reformule, encore une fois, d'une manière plus radicale: "Comment faire pour mettre en déroute, non pas les auteurs, mais la fonction de l'auteur, l'idée que derrière chaque livre il y a quelqu'un qui garantit la vérité de ce monde de fantasmes et fictions, par le seul fait qu'il y a investi sa vérité propre, qu'il s'est lui-même identifié avec cette construction de mots?" (p. 179).

Si l'on quitte Marana pour Flannery, le Journal de ce dernier nous indique qu'il est, lui aussi, favorable à la mort de l'Auteur. Sur ce point, il prolonge le constat que font les deux théoriciens. Roland Barthes rappelle, à juste titre, que ce sont les écrivains qui ont, les premiers, proclamé la mort de l'Auteur. Il cite Mallarmé, Valéry, Proust, les Surréalistes. Michel Foucault 
reprend le même argument: "L'œuvre qui avait le devoir d'apporter l'immortalité a reçu maintenant le droit de tuer, d'être meurtrière de son auteur. Voyez Flaubert, Proust, Kafka" (M.F., p. 793). Il s'agit, en fait, d'une re-formulation de l'idée selon laquelle il faut se passer de la biographie ou de la psychologie de l'auteur pour lire son œuvre. Roland Barthes développe ainsi: “[...] l'image de la littérature que l'on peut trouver dans la culture courante est tyranniquement centrée sur l'auteur, sa personne, son histoire, ses goûts, ses passions; [...] l'explication de l'œuvre est toujours cherchée du côté de celui qui l'a produite" (R.B., p. 62). Or, dans son journal, Silas Flannery semble reprendre à son compte le propos de Barthes, presque mot pour mot: «Le style, le goût, la philosophie, la subjectivité, la formation culturelle, et le vécu, la psychologie, le talent, les trucs du métier: tous les éléments qui font que ce que j'écris est reconnaissable, me semblent une cage qui restreint mes possibilités" (p. 19I). Ces repérages intertextuels font apparaître que, s'il y a bien réminiscence, ou paraphrase, des deux structuralistes français, Calvino ne leur réserve pas le même traitement. Marana soutient des thèses plus radicales que celles de Michel Foucault, sans doute parce qu'il doit beaucoup - son nom de consonance espagnole l'indique - à l'écrivain argentin, J.L. Borges. En revanche, même s'il emprunte des traits à Nabokov ou à Joyce, Silas Flannery développe une réflexion sur l'auteur essentiellement barthésienne.

Ces remarques partent d'une relecture de Calvino à la lumière de Barthes et Foucault, mais si l'on s'autorise l'inverse, en vertu du droit à l'anachronisme borgésien ou "menardien ", on découvre, à l'arrière-plan, ou comme ligne de fuite de leurs articles, la présence des figures paradoxales de l'auteur qui ont attiré notre attention chez Calvino. Michel Foucault évoque furtivement le cas du faussaire: "Dans le De viris illustribus, saint Jérôme explique que l'homonymie ne suffit pas à identifier d'une façon légitime les auteurs de plusieurs cuvres: des individus différents ont pu porter le même nom, ou l'on a pu, abusivement, emprunter le patronyme de l'autre " (M.F., p. 80I). L'article de Roland Barthes semble déployer un autre imaginaire puisque, à travers l'idée d'une littérature entièrement hypertextuelle, il se rapproche de la problématique du plagiat et qu'il évoque la figure du copiste: "[...] le texte est un tissu de citations, issu de mille foyers de la culture. Pareil à Bouvard et Pécuchet, ces éternels copistes, à la fois sublimes et comiques, et dont le ridicule désigne précisément la vérité de l'écriture, l'écrivain ne peut qu'imiter un geste toujours antérieur, jamais originel " (R.B., p. 65).

II. C'est moi qui souligne. 
En conclusion, la comparaison du roman avec les deux articles établit une intertextualité beaucoup plus riche et plus complexe que ne l'a montré la critique jusqu'à présent. Sur le fond, Italo Calvino ne souscrit pas à l'utopie barthésienne d'une mort de l'auteur débouchant sur la naissance du lecteur; en revanche, il rejoint certainement la conception, chère à Barthes, de l'écriture comme réécriture, à travers son intérêt pour la question du plagiat et, bien sûr, sa propre pratique du pastiche. Il reprend à Michel Foucault la problématique du changement d'attribution, mais il l'infléchit dans une optique borgésienne, en passant, notamment, à l'hypothèse d'une absence d'attribution. En définitive, nous aurions une sorte de "roman à clés", où Marana combine la figure de J.L. Borges et celle de Michel Foucault, tandis que Silas Flannery prend en charge celle de Roland Barthes, et nous avons une répartition de l'imaginaire qui lie Marana au faussaire et Flannery au plagiaire et au copiste. L'analyse intertextuelle met ainsi en évidence un rapport entre la mort de l'Auteur et ses figures paradoxales, rapport déjà présent en filigrane dans les articles de Barthes et Foucault. Cependant, ces figures n'y sont évoquées que furtivement. Sans doute parce que la mort de l'Auteur est d'abord, pour les deux théoriciens, une hypothèse de lecture et non pas un principe d'écriture. Ces derniers se demandent essentiellement comment et pourquoi lire des œuvres écrites par des auteurs, mais "décrochées" de l'intention de l'auteur par le lecteur.

\section{La mort de l'Auteur comme principe de lecture}

Le roman de Calvino illustre l'une des idées les plus importantes de Roland Barthes. Comme lui, il remet en cause une critique "tyranniquement centrée» sur l'Auteur, sa biographie, ses intentions. L'illustration se trouve essentiellement dans le "roman à lire" numéro 3 ("Penché au bord de la côte escarpée") et au chapitre $4 \mathrm{du}$ " roman à vivre " qui suit. Elle concerne l'interprétation de l'œuvre d'un auteur fictif, le Cimbre Ukko Ahti (p. 63-87). Le "roman à lire" se présente sous la forme du journal intime d'un jeune curiste, manipulé par une femme, pour qu'il l'aide à faire évader son complice d'une prison. Le récit multiplie les décalages entre le pessimisme, le sérieux du narrateur et son comique involontaire, son manque de bon sens. Il s'agit donc d'un texte finement comique, qui pastiche de grands romans de l'entre-deux guerres, tels que La Montagne magique de Thomas Mann ou La Nausée de Sartre. Telle est, en tout cas, la lecture à laquelle, nous, lecteurs, aboutissons. Or, au chapitre suivant, le professeur Uzzi Tuzii commente ce texte (p. 8I). Son commentaire suggère, au contraire, d'en faire une lecture 
tragique, à la lumière des données psycho-biographiques. L'inachèvement de l'œuvre devient implicitement la préfiguration de la mort prématurée de l'auteur, par suicide. En d'autres termes, Uzzi Tuzii, qui incarne le versant conservateur de l'Université, commet un contresens. Il ne peut admettre l'idée qu'un auteur déprimé puisse écrire un roman humoristique. Les rapports entre l'œuvre et la biographie peuvent donc être brouillés, par erreur, quand ils ne sont pas falsifiés, comme le suggère l'éditeur Cavedagna (p. III).

Un autre élément conforte cette critique des méthodes de lecture traditionnelles. Il faut, en effet, noter que le " retour à l'auteur ", opéré par certains personnages, est traité sur le mode comique. Ce retour à l'auteur, et non de l'auteur, a lieu au chapitre 8. Il est raconté par l'écrivain Silas Flannery, dans son journal. Il y a à la fois un comique de situation et un comique de répétition, puisque l'on voit défiler, successivement, Lotaria, Ludmilla et le narrataire, au chalet suisse de Flannery. Les visites de Lotaria et de Ludmilla reposent sur un comique de situation: la contradiction entre leurs théories respectives de la littérature et leur pratique. La visite du Lecteur était, en revanche, plus prévisible. Le narrataire appartient, en effet, à la catégorie des lecteurs primaires et traditionnels - tous ses goûts le trahissent - et l'on n'est pas surpris qu'il se tourne vers l'auteur pour faire authentifier l'un des deux romans fournis par Marana. Mais la scène tourne à la dérision lorsque Flannery, non content de refuser d'authentifier les livres, met en circulation un faux (p. 216-217).

On objectera qu'Italo Calvino se moque aussi des partisans de la mort de l'Auteur. La satire des habitués du séminaire de Galligani constitue la première étape (p. I03) ${ }^{\mathrm{I} 2}$, étroitement associée à celle du personnage de Lotaria et de ses doubles, incarnant une certaine hystérie révolutionnaire et féministe (ch. 8, 9, 10). Non sans humour, le narrateur commente la visite du narrataire à la maison d'édition:

[...] c'est le moment (dans l'histoire de la culture occidentale) où ceux qui cherchent à se réaliser sur du papier ne sont plus des individus isolés mais des collectivités: séminaires d'études, groupes de recherche, équipes, comme si le travail intellectuel était trop désolant pour pouvoir être affronté dans la solitude. La figure de l'auteur est devenue plurielle, et se déplace toujours en groupe parce que - en plus - personne ne peut représenter personne: quatre ex-détenus dont un évadé, trois ex-hospitalisés avec leur infirmier et le manuscrit de ce dernier. (p. 108)

I2. Pour une analyse plus précise de la satire des courants de la Nouvelle Critique, voir Anne-Marie Monluçon, "Critique et fiction. Petits Meurtres entre amis", Recherches of Travaux, $\mathrm{n}^{\circ}$ 60, Université Stendhal-Grenoble 3, 2002, p. I23-I42. 
Ce passage pointe les contradictions d'une époque dont on se demande si elle veut la mort de l'Auteur ou la démocratisation de son statut. Le collectif ne serait-il pas le masque d'un retour de l'Auteur? Est-ce le statut d'auteur ou celui d'écrivain qui fascine? Le récit jette aussi un certain discrédit sur les deux partisans les plus explicites de la mort de l'Auteur: Marana et Flannery. En premier lieu, Marana est disqualifié par une motivation basse. Poussé par la jalousie, il veut faire écran à la relation qu'entretient Ludmilla, la lectrice, avec Silas Flannery (p. 178). Ensuite, Silas Flannery est ridiculisé par les contradictions entre sa pratique et sa théorie. Sa tentative de séduction de Ludmilla prouve que, bien qu'il prône l'effacement de l'auteur, il ne veut pas renoncer aux avantages que confere le statut d'écrivain. En outre, sa motivation est aussi suspecte, car son désir d'effacement survient en même temps qu'une panne d'inspiration (p. 137). Ces traits ne sont peut-être que la parodie des arguments des adversaires de la mort de l'Auteur. En revanche, la critique, toujours implicite, devient sérieuse, si l'on prend garde au fait que Silas Flannery, qui prétend vouloir s'effacer, tient son journal, à propos duquel il se demande si ce n'est pas cela son "vrai livre", le chef-d'œuvre qu'il ne parvient pas à écrire depuis des années (p. 20I) ${ }^{\text {I3 }}$. Il paraît en effet difficile de prôner la mort de l'Auteur et de cultiver le genre autobiographique.

Si l'on essaie de dégager le point de vue de l'auteur Calvino sur la question (présupposé évidemment condamné par la théorie de la mort de l'Auteur), il faut sans doute le chercher à trois niveaux: dans les moments de consensus, dans les personnages les plus nuancés, dans la réflexion sur les limites des substitutions possibles à l'Auteur. La thèse de la mort de l'Auteur, comme principe de lecture, est d'abord étayée par un moment de consensus entre Marana et l'éditeur Cavedagna. Ce dernier donne presque raison au faussaire, parce que celui-ci développe l'argument historique, le plus facile à accepter, selon lequel on peutse passer de la fonction-auteur, parce qu'on s'en est deja passé, au temps d'Homère, de Socrate, bien avant la naissance de l'Auteur, au sens moderne: «-Vous avez vu le beau raisonnement? s'exclame Cavedagna. Le pire, c'est qu'il pourrait bien avoir raison..." (p. II4). Le second moment de consensus figure au chapitre 8. A deux reprises, Silas

13. Il n'est d'ailleurs pas exclu qu'il s'agisse aussi d'une critique ad hominem, car Roland Barthes s'est beaucoup contredit (M. Couturier, La Figure de l'Auteur, Seuil, 1995, p. 13, et Roland Barthes, Plaisir du texte, p. 45-46). Bien qu'il n'ait jamais osé franchir explicitement le pas, entre critique et création, il a tout de même publié trois textes de nature autobiographique (Roland Barthes, Seuil, coll. "Écrivains de toujours", 1975, Fragment d'un discours amouretux, Seuil, coll. "Tel Quel», 1977, La Chambre claire. Notes sur la photographie, Seuil, coll. "Cahiers du cinéma ", 1980). 
Flannery note, dans son journal, son accord avec Marana (p. 20I et 2I4) : "Je voudrais retrouver Hermès Marana pour lui proposer de nous associer et d'inonder le monde d'apocryphes" (p. 2I4). L'écrivain irlandais opère, au passage, un saut qualitatif, puisqu'il fait de la mort de l'Auteur un principe de production des textes. Reste à savoir s'il s'agit d'une thèse sérieuse (borgésienne) ou d'une hypothèse comique, pour amener chacun à réfléchir aux conséquences de la mort de l'Auteur, si elle déborde le cadre de la lecture.

Sur un autre plan, Cavedagna incarne, avec Ludmilla, la fraction éclairée des lecteurs traditionnels, dont la conception de l'Auteur n'est guère contestée. Tous deux font une distinction très nette entre l'Auteur et sa personne: "[...] pour Ludmilla les auteurs ne s'incarnent jamais dans des individus de chair et d'os, ils n'existent pour elle que dans les pages publiées, vivants ou morts, [...] et elle est prête à les suivre, avec cette légèreté volubile qu'on a dans les rapports avec des personnes incorporelles" (p. 179, voir aussi p. 212). L'éditeur souscrit même à l'idée, familière aux borgésiens, selon laquelle l'Auteur est une fiction au même titre que les personnages:

[...] les auteurs véritables restent ceux qui n'étaient pour lui qu'un nom sur une couverture, un mot qui ne se laissait pas séparer du titre, des auteurs qui partageaient la réalité de leurs personnages ou des lieux nommés dans les livres, qui existaient et en même temps n'existaient pas, comme les personnages et les lieux. L'auteur était sur le point invisible d'où partaient les livres, un vide parcouru de fantômes [...] (p. II4).

Malgré une audace toute borgésienne, Italo Calvino pose peut-être une limite. L'une des substitutions proposées à l'Auteur n'est ni le "nègre", ni le faussaire, mais l'ordinateur. Silas Flannery fait l'expérience d'être lu par ordinateur (p. 207-209) et celle d'imaginer que l'ordinateur le remplace pour écrire. La lecture électronique donne un résultat assez ridicule: le texte est réduit à une liste de mots, "pronoms, particules" (p.209, 244). Cette méthode écarte, évidemment, l'intention de l'auteur, mais aussi la subjectivité du lecteur. Le comique condamne ici la déshumanisation d'une telle lecture. L'hypothèse inverse - peut-on remplacer non plus le lecteur mais l'Auteur? - par l'ordinateur est l'objet d'une conclusion moins nette: «Peutêtre qu'au lieu d'écrire un livre, je pourrais dresser des listes de mots par ordre alphabétique, une cascade de mots isolés où s'exprimerait la vérité que je ne connais pas encore, et à partir desquels l'ordinateur, en retournant son programme, obtiendrait un livre: mon livre" (p. 209). Avec l'ordinateur, Italo Calvino semble fixer une limite aux substitutions: la mort de l'Auteur s'arrête là où commenceraient à régner déconstruction et déshumanisation. Sa réflexion, présentée sur le mode ludique, est pourtant sérieuse, si l'on en croit l'article de Jonathan Usher ${ }^{14}$. Celui-ci indique qu'Italo Calvino a d'abord 
fondé certains espoirs sur l'écriture par ordinateur, car il considérait que beaucoup d'histoires résultent de la combinatoire d'éléments qui varient assez peu. Mais, entre 1967 et 198I, sa confiance dans les capacités de la machine s'est nettement réduite.

En définitive, Italo Calvino reprend à son compte la remise en cause du lien automatique entre l'ouvre et l'auteur comme principe de lecture. Il nuance cependant la thèse des théoriciens par l'humour et s'en écarte sur deux points. En effet, comment expliquer que, dans son roman, ceux qui lisent soient hostiles à la mort de l'Auteur, tandis que ceux qui écrivent y sont favorables? À l'inverse de Roland Barthes qui assimile systématiquement l'Auteur à la catégorie des oppresseurs (bourgeois, roi, Dieu) et considère sa "mort» comme le préalable nécessaire à la naissance du lecteur - ou mieux, à sa libération -, Italo Calvino révèle, par la distribution des rôles dans son roman, que le vrai conflit ne se situe pas entre l'Auteur, monarque tyrannique, et les autres, mais entre le lecteur primaire, traditionnel, qu'incarne le narrataire, et les autres (Auteurs, avant-gardes de la critique, et lecteurs éclairés, comme Ludmilla ou Cavedagna). Deux indices trahissent ce clivage: d'abord, la satire dont le narrataire est l'objet, et, ensuite, le retour en force du contrôle de l'auteur sur le lecteur réel, que permet, par ricochet, la constitution du narrataire comme figure repoussoir. Avec Michel Foucault, il s'agit moins d'une divergence que d'un dépassement, puisque le récit calvinien pousse l'hypothèse du changement d'attribution des textes jusqu'à son idéal borgésien: la production d'une littérature anonyme, à l'initiative du faussaire. C'est exactement là que la mort de l'Auteur, de principe de lecture qu'elle était, devient principe d'écriture.

\section{La mort de l'Auteur comme principe d'écriture}

C'est en explorant la possibilité de produire une littérature sans auteur qu'Italo Calvino introduit les figures paradoxales qui nous intéressent. Son roman propose un éventail à peu près complet de tous ceux qui écrivent sans jouir du statut d'Auteur: le traducteur, les "nègres" ou ghostwriters, et, bien sûr, le faussaire, le plagiaire, voire le copiste et l'ordinateur. Certains de ces substituts incarnent des pratiques littéraires illégales et le récit calvinien insiste assez nettement sur les problèmes juridiques et économiques que

14. Jonathan Usher, "Calvino and the Computer as Writer/Reader", The Modern Language Review, Leeds, England, MLR, I995 Jan: I, p. 4I-54. 
poserait la remise en cause de l'Auteur comme principe de production des textes.

Par ordre d'apparition, Marana le faussaire est la première figure paradoxale du roman. Ce personnage est mû par des motivations hétérogènes: d'un côté, le désir de perturber les relations entre Ludmilla et Silas Flannery, et, de l'autre côté, l'ambition beaucoup plus théorique d'entreprendre une mystification généralisée, dans le domaine de la littérature (p. I13, 179, 200, théorie reprise par l'APO, p. 146). Son existence même démontre la fragilité du lien entre l'auteur et l'œuvre, puisque celui-ci peut-être falsifié. Il apparaît d'abord comme un faussaire, parce qu'il fait passer la traduction d'un roman belge pour celle d'un roman cimbre, puis polonais. L'éditeur Cavedagna, qui l'a démasqué, l'accuse ensuite de plagiat (p. II4), parce qu'il a produit, semble-t-il, une traduction non autorisée du Belge Vandervelde ${ }^{15}$. Son troisième délit consiste à proposer une "option " sur la traduction en italien d'un roman de Silas Flannery dont une maison d'édition cimmérienne, installée en Amérique du Sud, a les droits en exclusivité. L'invraisemblance de ce montage éditorial suggère soit qu'il monnaye des droits de traduction qu'il ne possède pas, soit qu'il tente, par ce biais, de mettre en circulation un faux Flannery (p. 133). Marana est donc faussaire et plagiaire.

Il ne se contente pas de s'immiscer entre textes et auteurs, il vise aussi à dégrader - au nom d'une véritable théorie littéraire, rapportée par Silas Flannery dans son journal - la figure de l'Auteur. "[...] d'après lui, la littérature ne vaut que par son pouvoir de mystification, et ne trouve que dans la mystification sa vérité: un faux, en tant que mystification d'une mystification, est en somme une vérité à la puissance deux" (p. 200). Une partie de son activité consiste aussi à inverser les rôles entre lui et Silas Flannery, en suggérant que c'est l'auteur Silas Flannery qui est un plagiaire. Il commence par introduire l'idée que tous les auteurs seraient les plagiaires "d'un vieil Indien appelé le "Père des récits" ».

On a pu vérifier que nombre de romans publiés par des auteurs fameux avaient été racontés mot pour mot par la voix catarrheuse du *Père des Récits " plusieurs années avant leur sortie. Le vieil Indien serait, selon certains, la source universelle de la matière narrative, le magma primordial d'où partent les manifestations individuelles de chacun de ceux qu'on nomme écrivains [...] (p. 133).

L'accusation ne s'impose pas d'emblée, car Silas Flannery résiste d'abord à plusieurs tentations. Durant la période où le manque d'inspiration le paralyse, il refuse l'aide des nègres, que lui offre la société qui gère sa carrière

I5. Hélène Maurel-Indart, Du plagiat, 1999, PUF, coll. « Perspectives critiques », p. 174. 
(p. 137$)^{16}$, puis celle d'un ordinateur que lui propose Marana, pour achever le roman qu'il s'est engagé à écrire (p. 138). Ce dernier passe alors à l'offensive. Il se présente comme le défenseur des "intérêts d'un écrivain belge, Bertrand Vandervelde, impudemment plagié par Flannery" (p. 139) ${ }^{17}$. Puis il offre ses services à l'écrivain irlandais, pour le défendre des contre-façons japonaises (p. 199). A ce stade de l'analyse, ce ne sont que les insinuations et accusations sans preuve d'un personnage, Marana, qui est en partie disqualifié par la jalousie qui le motive, et dont les histoires, pleines d'invraisemblances, portent à le soupçonner de mythomanie. Dans ces conditions, le lecteur réel vat-il tenir compte de ses allégations? en fait, ce qui est décisif, c'est que la narration semble donner raison à Marana.

Les véritables tentations de l'auteur de thrillers ont, en fait, une source intérieure. Silas Flannery cherche à surmonter son manque d'inspiration par un exercice de copie:

Aujourd'hui je vais tenter de recopier les premières phrases d'un roman célèbre, pour voir si la charge d'énergie contenue dans ce début se communique à ma main [...]. Je m'arrête avant d'être submergé par la tentation de recopier Crime et Châtiment en entier. Pendant un instant, je crois comprendre ce qui a dû être le sens et l'attrait d'une vocation désormais inconcevable: celle du copiste. (p. 198-199)

Or, comme l'explique Hélène Maurel-Indart, il suffirait d'ôter les guillemets qui encadrent la citation du roman de Dostoïevski pour que la copie devienne plagiat (p. 173) ${ }^{18}$. Par petites touches, la narration suggère que le faussaire serait, en fait, un auteur inspiré et productif, et que l'auteur tourmenté finit par lui voler ses idées et le plagier. La démonstration de ce renversement nécessite un petit détour. Il faut savoir que Marana a inventé, pour satisfaire une lectrice princière, la femme d'un sultan, une œuvre qui emprunte sa structure aux Mille et Une Nuits, ainsi que l'explique sa correspondance, parcourue par le héros lecteur:

Bref, Marana a proposé au sultan un stratagème inspiré de la tradition littéraire de l'Orient: il interrompra sa traduction au moment le plus passionnant, et commencera à traduire un autre roman, en l'insérant dans le premier par

I6. Les nègres sont une catégorie tolérée, sinon licite, de plagiaires (ils sont experts dans l'art d'imiter le style du maître) et faussaires (ils ne signent pas leur production).

17. Les circonstances du plagiat se précisent: Silas Flannery voudrait faire de l'écriture une "lecture de la lecture", trouver l'inspiration sur le visage de la lectrice, que l'on soupçonne d'être Ludmilla (p. 144). En clair, il voudrait écrire un roman proche de celui qui la captive si intensément. Par malchance, cela le placerait sous l'influence de la médiocre lecture de cette femme, un roman de "quatre sous", du Belge Vandervelde.

18. H. Maurel-Indart, op. cit., p. 173. 
quelque expédient rudimentaire, par exemple un personnage du premier roman ouvre un livre et se met à lire... (p. 14I)

Ce procédé - véritable mise en abyme de la structure de Si par une nuit d'hiver un voyageur - revient à ne donner à lire à la lectrice que des débuts de roman. Or, Silas Flannery reçoit la visite du héros lecteur, qui lui communique, sans le savoir, l'idée de Marana (p. 2I8) ; aussitôt après, Silas Flannery note dans son journal: «L'idée m'est venue d'écrire un roman tout entier fait de débuts de romans" (p. 219). En d'autres termes, Silas Flannery plagie, sans le savoir, l'idée de Marana.

La narration s'emploie à démontrer ainsi que celui qui est un faussaire sur le plan juridique est, peut-être, sur le plan littéraire, un véritable auteur, parce qu'il est créatif. On pourrait objecter qu'il doit persister une différence qualitative entre l'auteur et le faussaire. Mais l'écrivain irlandais prévient luimême cette objection. Il fait l'hypothèse que certaines contrefaçons peuvent constituer de vraies œuvres: " moyennant quoi [...] les faux de Silas Flannery [...] peuvent en même temps receler une sagesse raffinée, secrète [...]" (p. 199). Cette prise de position parachève la déstabilisation des frontières entre auteur et faussaire, en confirmant le renversement, lorsque Silas Flannery avoue, dans son journal, "sa fascination" pour les faux: "Je voudrais retrouver Hermès Marana pour lui proposer de nous associer et d'inonder le monde d'apocryphes» (p. 2I4). L'auteur pourrait donc être tenté non seulement par le plagiat, mais par l'écriture apocryphe.

L'analyse intertextuelle a montré que le rapport entre la mort de l'Auteur et ses figures paradoxales existe déjà dans les articles de Roland Barthes et Michel Foucault, avant d'être développé par Calvino. Ces figures posent implicitement la question des conséquences ultimes de la théorie: peut-on penser la mort de l'Auteur comme principe de lecture sans la penser aussi comme principe d'écriture? L'articulation de la lecture à l'écriture comble précisément l' «impensé" des analyses de Barthes et Foucault, et elle coïncide, vraisemblablement, avec la dette de Calvino envers Borges, l'un des premiers écrivains, sans doute, à concevoir et souhaiter l'avènement d'une littérature anonyme.

Si non seulement les personnages d'auteur, tels que Silas Flannery, mais aussi les auteurs réels, comme Calvino et Borges, sont tous favorables à la mort de l'Auteur, quelle incidence a donc le changement qui consiste à passer du point de vue des théoriciens à celui des auteurs? En premier lieu, le parti pris, très humoristique, de Calvino, a pour effet de désamorcer la polémique, lancée, non sans un certain sens de la dramatisation, par Roland 
Barthes. En second lieu, il la déplace, en montrant, à travers la figure du narrataire, qui n'est autre qu'un modèle de mauvais lecteur, que la mort de l'auteur, entendue comme principe de lecture, refusant essentiellement de réduire le texte à la psycho-biographie ou aux intentions de l'auteur, n'a pas pour vocation de déranger les auteurs - consentants, pour la plupart - mais un certain type de lecteur: traditionnel, ne s'intéressant quasiment qu'à l'intrigue, ce qui explique son attachement à la lecture intégrale et linéaire, et son intérêt exacerbé pour le dénouement.

Cette réflexion critique sur la lecture constitue, par un renversement assez spectaculaire, un retour non pas à l'Auteur, mais de l'Auteur, au sens où celui-ci exerce, à travers la satire du lecteur fictif, un contrôle indirect sur le lecteur réel ${ }^{19}$. Le jeu d'apparitions/disparitions, auquel se livre Italo Calvino dans son roman, en est peut-être l'aveu ${ }^{20}$. Nous avons aussi montré que les intentions de l'auteur se laissent décrypter à travers les figures de lecteurs éclairés, que sont Cavedagna et Ludmilla, beaucoup plus épargnés par la satire, ainsi que dans les moments de consensus. Parmi ceux-ci, le plus étonnant est l'accord de l'auteur, Silas Flannery, avec le faussaire, Marana, pour «inonder le monde d'apocryphes». Le consensus porte alors sur la mort de l'Auteur comme principe d'écriture. Cette idée permet un second retour de l'Auteur, nọn plus comme critique guidant son lecteur, mais comme créateur. En effet, si, dans un premier temps, Marana ne fait que parachever l'entreprise de démolition commencée par les théoriciens, dans un second temps, lorsque la narration a établi la supériorité de Marana sur Flannery en matière de création, le faussaire devient peut-être le masque d'un retour ou d'une résurrection de l'Auteur, un auteur auquel il ne manque qu'une signature et un statut. Le renversement spectaculaire des rôles entre l'auteur et le faussaire indique que la réflexion juridique - qu'est-ce qui est licite? - n'est qu'un détour, avant de revenir au plan littéraire - qu'est-ce qui est créatif?

À ce niveau, Calvino rappelle avec insistance la nature profondément ambiguë de la littérature, ce qui fait de l'Auteur une instance insaisissable. Non seulement, comme le suggère la dialectique des relations entre les per-

19. Sur ce point, je m'écarte de l'analyse de C. Segre. Voir supra note 9.

20. Au premier chapitre, le narrateur présente Italo Calvino comme l'auteur du chapitre suivant, intitulé "Si par une nuit d'hiver un voyageur". Mais ce chapitre est ensuite attribué à un auteur fictif, le Polonais Tadzio Bazabkal. L'auteur Calvino ne reparaît alors qu'au dernier chapitre, à moins qu'on ne lui attribue l'un ou l'autre des "romans à lire * qui sont restés sans attribution (le sixième ou le septième). Au dénouement, Italo Calvino est désigné comme l'auteur du roman que termine le héros, sans que l'on sache précisément quelle est l'étendue de l'œuvre qu'on lui attribue. À cet endroit, le nom fonctionne un peu comme une signature. 
sonnages, le faussaire peut être un créateur plus authentique que l'auteur, mais, comme le démontre activement Calvino, par sa pratique du pastiche et de la parodie, dans les dix «romans à lire", ainsi que par le jeu de l'auto-citation ${ }^{2 I}$, il n'est peut-être pas de littérature créative qui ne soit réécriture, c'està-dire pratique de l'emprunt, toujours voisine du plagiat. Sa réflexion sur l'Auteur comme créateur prolonge celle qu'a inaugurée Borges dans la célèbre nouvelle "Pierre Menard, auteur du Quichotte "22", en reprenant ses conclusions et en se demandant aussi comment écrire après le coup de génie de l'écrivain argentin. Calvino propose deux variations sur la trouvaille de Borges dans son "Pierre Menard". La première, réussie, consiste en une multiplication virtuose du mécanisme du changement d'attribution - employé en hapax dans la nouvelle -, avec une variation sur les causes d'un tel changement et sur sa présentation - Borges l'annonce, Calvino le révèle après coup. La seconde est une parodie, soit, ici, un hommage sous la forme d'un échec volontaire. Il s'agit des pages du journal de Silas Flannery où celui-ci recopie le début de Crime et Châtiment, en espérant de la sorte retrouver l'inspiration (p. 198). Mais cette copie, conçue comme stimulation et non pas recréation, ne produit aucun effet, sinon comique. Par ces deux exemples, Calvino redéfinit peut-être ce qu'est un créateur authentique: un esprit à la fois totalement dépourvu d'imagination (Menard reproduit le début de Don Quichotte) et un inventeur de génie (Borges exploite les potentialités d'un changement d'attribution, du fonctionnement du "nom d'auteur"); pour la postérité (Calvino), il est à la fois merveilleusement fécondant, puisqu'il réemploie sa trouvaille, et parfois stérilisant, inimitable, certains coups de génie étant voués à l'hapax. En d'autres termes, la mort de l'Auteur interdit de réduire la création à l'originalité.

Le roman de Calvino nous offre ainsi le paradoxe d'un retour de l'Auteur dans un roman qui prétend s'en passer. Mais il ne s'agit ni d'un retour en arrière, ni d'un retour à l'identique. L'auto-ironie serait sa manière de prendre en compte les acquis de la critique, et la subversion serait sa manière de redevenir maitre du jeu.

21. Italo Calvino pratique l'intratextualité et parvient ainsi à être présent dans son roman, grâce aux références à ses œuvres. Le sixième "roman à lire" s'intitule "Dans un réseau de lignes entrelacées * et le septième "Dans un réseau de lignes entrecroisćes", ce qui n'est pas sans rappeler le roman de Calvino Le Chateau des destins croisés (1973), trad. de l'italien par J. Thibaudeau et l'auteur (1976) Seuil, Points. Roman, 1988. En outre, lorsqu'il évoque l'étude lexicale par ordinateur, menée par Lotaria, il cite en note les références d'une véritable étude universitaire (p. 209), sans signaler que le texte qui a servi de support est celui de son roman Le Sentier des nids d'araignée (1947, traduit en français en 1978, chez Julliard).

22. J.L. Borges, Fictions, (1944), trad. de l'espagnol par R. Caillois, N. Ibarra et P. Verdevoye, revue par J.-P. Bernès, in Euvres complettes, t. I, Paris, Gallimard, coll. "Bibliothèque de La Pléiade", 1993. "Pierre Menard " date de 1939. 